\title{
Neuroparacoccidioidomicosis: para tener presente
}

\author{
Recibido: 28/11/18 Aceptado: 23/2/19
}

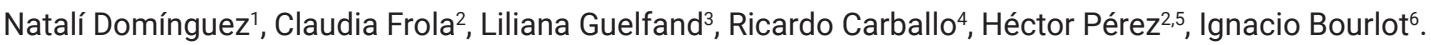

\section{Resumen}

La paracoccidioidomicosis (PCM) es una micosis endémica de zonas tropicales y subtropicales, con mayor prevalencia en América Latina, producida por especies del género Paracoccidioides. Es una micosis profunda sistémica que en su forma crónica afecta principalmente a varones adultos. La afectación del sistema nervioso central (SNC) está descrita en un $10-27 \%$. Nosotros presentamos dos casos con manifestación neurológica al ingreso.

Palabras clave: paracoccidioidomicosis, neuroparacoccidioidomicosis, sistema nervioso central.

\section{Introducción}

La paracoccidioidomicosis (PCM) representa la micosis de mayor prevalencia en América Latina. Se trata de una micosis profunda sistémica y endémica más frecuente en inmunocompetentes, producida por especies del género Paracoccidioides. En 2009 se identificaron dos especies dentro del género: $P$. brasilensis, integrada por cuatro especies crípticas denominadas S1, PS2, PS3 y PS4, y P. lutzii. Actualmente se propuso elevar estas cuatro especies crípticas a especies taxonómicas. De este modo, los agentes causales son: $P$. brasilensis (S1), P. americana (PS2), P restrepiensis (PS3), $P$. venezuelensis (PS4) y el ya identificado $P$. lutzii $(1,2)$.

Se la encuentra en áreas tropicales y subtropicales de América Latina, con Brasil como principal país endémico, y en menor porcentaje Venezuela, Colombia, Argentina y Ecuador. Nuestro país presenta dos áreas endémicas, las provin-
${ }^{1}$ Servicio de Clínica Médica, Hospital J. J. Urquiza $C$ del Uruguay, Entre Ríos, Argentina. ${ }^{2}$ División Infectología, HGA Dr. Juan A. Fernández, CABA, Argentina. ${ }^{3}$ Laboratorio de Microbiología, HGA Dr. Juan A. Fernández, CABA, Argentina. ${ }^{4}$ Laboratorio de Microbiología, Hospital J. J. Urquiza, C. del Uruguay, Entre Ríos, Argentina. ${ }^{5}$ Docente Adscripto, Universidad de Buenos Aires, orientación infectología, CABA, Argentina. ${ }^{6}$ Médico consultor infectología, Hospital J. J. Urquiza, C. del Uruguay, Entre Ríos, Argentina.

Ninguno de los autores declara presentar conflicto de intereses en relación a esta publicación.

Dirección para correspondencia:

Natalí Domínguez, Servicio de Clínica Médica. Hospital J.J. de Urquiza. Av. Uncal s/n. 3260. C. del Uruguay. Entre Ríos. Argentina. Tel: (03442) 443900 Int 306 E-mail: domingueznataliy@gmail.com

cias del Noreste (Chaco, Corrientes, Misiones y Formosa), donde se da el $85 \%$ de los casos, y las provincias del Noroeste (Salta, Jujuy y Tucumán) (3).

La primoinfección suele ser asintomática y tiende a resolverse espontáneamente. Más del $95 \%$ de los casos que se presentan en la edad adulta son secundarios al largo periodo de latencia.

La agresividad del hongo y el perfil inmunológico del huésped permiten observar diferentes formas clínicas de presentación. Entre ellas se mencionan la forma aguda/suba- 
guda o juvenil que se manifiesta en niños y adolescente y la forma crónica o adulta, que representa el $90 \%$ de los casos. Esta última presentación se observa en varones, de entre 30 y 60 años, e involucra más de un órgano simultáneamente, siendo el compromiso pulmonar y cutáneo-mucoso los más frecuentes.

El compromiso del sistema nervioso central (SNC) está descrito en un 10-27\% (4), aunque su presentación clínica inicial es rara e inusual.

A continuación se presentan dos casos con manifestación neurológica al ingreso.

\section{Caso 1}

Masculino de 61 años, de Oberá (Misiones). Leñador. Tabaquista y estilista de jerarquía.

Ingresa por cuadro de astenia, disfagia e hiporexia de un mes, con alteración del sensorio en las últimas semanas.

Examen físico en regular estado general. Temperatura $37,8^{\circ}$ C, frecuencia respiratoria 20 por minuto y saturación de $\mathrm{O}_{2}$ 96\% a/a. Fauces congestivas y eritematosas, con exudado amigdalino. Úlcera en paladar duro y velo de paladar. Adenopatías cervicales bilaterales múltiples, indoloras y duro-pétreas. Laboratorio: hemoglobina $10,5 \mathrm{~g} \%$, plaquetas $522.000 / \mathrm{mm}^{3}$, leucocitos $13.200 / \mathrm{mm}^{3}$, glucemia, función hepática y renal normales. Sodio $126 \mathrm{mEq} / \mathrm{l}$. Eritrosedimentación: 35 mm. Albúmina 3,4 g/dL.

Tomografía Computada (TC) de encéfalo: sin lesión ocupante de espacio ni sangrado. TC de tórax presencia de opacidades en vidrio esmerilado, de distribución periférica y basal, con engrosamiento de septos interlobulillares.

Por ausencia de imagen patológica de encéfalo e hiponatremia al ingreso se interpreta la alteración del sensorio de causa metabólica.

Hemocultivos para gérmenes comunes, esputo con cultivo para gérmenes comunes y B.A.A.R en tres muestras negativos. Lavado bronco alveolar con examen en fresco y cultivo negativo.

Escarificación de lesión en región palatina con examen directo y coloración de Giemsa con elementos levaduriformes compatibles con Paracoccidioides spp.
Serologías para $\mathrm{VIH}$, sífilis, hepatitis $\mathrm{B} / \mathrm{C}$, toxoplasmosis, Chagas, estudio de mononucleosis, anti-Leishmania IgG no reactivas. Inmunodifusión de Paracoccidioides positiva.

Con diagnóstico confirmado de PCM se inició tratamiento con itraconazol, 200 mg por día, por inmediata disponibilidad y buena tolerancia a la vía oral. En la primera semana evoluciona con inestabilidad en la marcha y ataxia, por lo que se realiza a las 24 hs siguientes una resonancia magnética (RM) de encéfalo con contraste, que informa múltiples lesiones en fosa posterior y en áreas corticales. Lesión expansiva subcallosa extendida hacia cuerno frontal izquierdo. Por probable compromiso de SNC por Paracoccidoides se rota antifúngico a anfotericina $\mathrm{B}$ desoxicolato con mala evolución clínica y óbito luego de nueve días de instaurado.

\section{Figura 1. RNM de encéfalo con contraste}

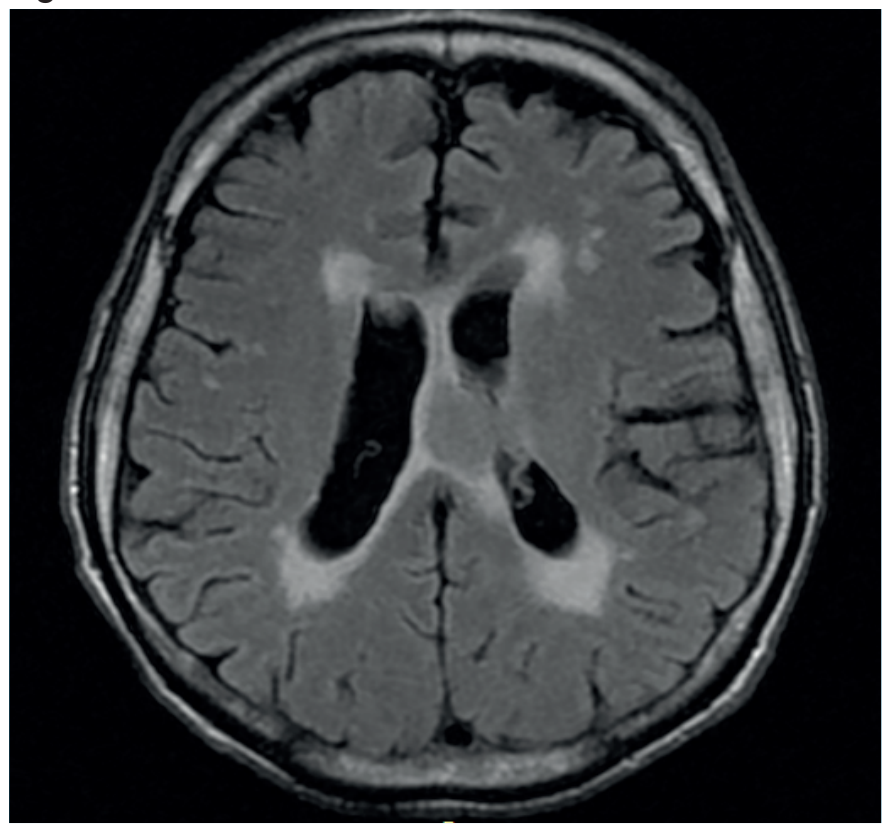

Se observa lesión expansiva subcallosa extendida hacia cuerno frontal izquierdo.

\section{Caso 2}

Masculino de 37 años, oriundo de Paraguay, residencia en ciudad de Buenos Aires desde hace 20 años. Trabajador de aserradero. Tabaquista severo y enolista.

Ingresa por un cuadro de cefalea holocraneana y visión borrosa de dos meses.

Examen físico en buen estado general. Afebril, frecuencia respiratoria 16 por minuto y saturación de $\mathrm{O}_{2} 96 \%$ a/a. Hemianopsia temporal izquierda, sin otros hallazgos de relevancia. 
Laboratorio: hemoglobina $15,4 \mathrm{~g} \%$, plaquetas $264.000 / \mathrm{mm}^{3}$, leucocitos $18.300 / \mathrm{mm}^{3}$, glucemia, ionograma, función hepática y renal normales. Eritrosedimentación: 75 mm. Albú$\operatorname{mina} 3 \mathrm{~g} / \mathrm{dL}$.

TC y RM de encéfalo: lesión ocupante de espacio occipital derecha con realce periférico al contraste y edema perilesional.

Serologías para VIH y Chagas no reactivas.

Se interna en servicio de neurocirugía para biopsia cerebral.

Radiografía de tórax al ingreso con opacidad parahiliar derecha de bordes mal definidos, por lo que se solicita estudio complementario específico.

TC de tórax con múltiples nódulos de contornos lobulados, algunos cavitados, en ambos lóbulos superiores. Parches de vidrio esmerilado. Nodulillos centrolobulillares con patrón de "árbol en brote".

Esputo para gérmenes comunes y B.A.A.R en tres muestras negativos. Lavado bronco alveolar con examen en fresco y Giemsa con levadura esférica multibrotada compatible con Paracoccidiodes spp. Inmunodifusión de Paracoccidiodes spp. positiva. Espirometría con alteración ventilatoria obstructiva de carácter moderado.

En contexto de descartar otra inmunosupresión diferente al VIH se solicita recuento de células T CD4+ con un valor de 952 (32\%), dentro de los parámetros de normalidad.

Con diagnóstico probable de PCM diseminada con compromiso de SNC se inicia tratamiento con anfotericina $B$ desoxicolato. Evoluciona a la semana del mismo con disnea, fiebre persistente y desaturación. Nueva TC de tórax con progresión de infiltrados parenquimatosos y derrame pleural bilateral y TC de abdomen sin hallazgos patológicos. Ausencia de adenomegalias. Toracocentesis con exudado no complicado. Hemocultivos para gérmenes comunes, micobacterias y hongos negativos.

Se interpreta como probable "reacción paradojal" asociada a PCM y se adiciona al tratamiento antifúngico, trimetoprima-sulfametoxazol (TMS) más corticoterapia con buena respuesta clínica e imagenológica a nivel pulmonar. Luego de 30 días de biterapia, se suspende anfotericina B sin complicaciones.
RM de encéfalo a las seis semanas de tratamiento sin cambios significativos.

Para descartar patología concomitante se realiza exéresis de la lesión con biopsia por congelación compatible con Paracoccidiodes spp. e informe definitivo de absceso micótico.

Tras dos meses de internación y buena evolución clínica se decide continuar tratamiento ambulatorio con TMS, el cual completa por 27 meses. Serologías para Paracoccidiodes spp. posteriores al inicio de la terapia negativas en múltiples oportunidades.

Actualmente con mejoría de la alteración visual con último control por imágenes de SNC que evidencia lesión secuelar. Patrón obstructivo leve como secuela pulmonar. Continua con controles serológicos y clínicos de manera multidisciplinaria cada seis meses durante el primer año libre de tratamiento.

\section{Figura 2. RNM de encéfalo con contraste}

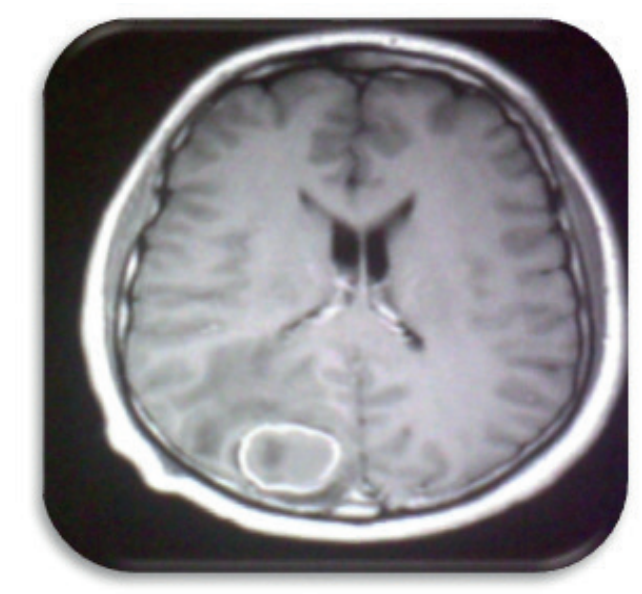

Se observa lesión ocupante de espacio occipital derecha con realce periférico al contraste y edema perilesional

\section{Discusión}

La PCM es la micosis sistémica más prevalente en países de América Latina. Su distribución está limitada a regiones subtropicales de Latinoamérica, con el norte de nuestro país como área endémica.

El nicho ecológico de Paracoccidioides permanece indefinido, aunque el hábitat medioambiental de la fase micelial es el suelo, donde se encuentran las estructuras infectantes (5). 
Afecta tanto a huéspedes inmunocomprometidos como inmunocompetentes. Este último escenario corresponde a los casos clínicos presentados. Sin embargo, se plantean otros factores que pueden estar asociados con el desarrollo de esta micosis, como por ejemplo la desnutrición. En este sentido, según los valores de albúmina de ingreso de ambos pacientes, los mismos presentaban un cuadro de desnutrición leve. Adicionalmente se han hallado al tabaquismo y al alcoholismo como factores asociados, ambas condiciones comunes en los casos detallados.

La PCM es más prevalente en trabajadores agrícolas en áreas rurales (6) debido probablemente a la forma micelial del hongo que se desarrolla a temperatura ambiente en la tierra, en el agua y en las plantas $(6,7)$. En ambos pacientes presentados la exposición laboral podría ser predisponente.

La primoinfección suele ser asintomática en la mayoría de los huéspedes inmunocompetentes y tiende a resolverse en forma espontánea. Sin embargo, puede pasar a un estado de latencia cuya duración depende de la respuesta inmunitaria, aunque en la edad adulta más del 95\% de los casos son secundarios a largos periodos $(7,8)$.

La PCM se puede presentar como una enfermedad progresiva aguda o subaguda, denominada forma infanto-juvenil o como una forma crónica, que es la presentación más frecuente. Si bien el segundo caso clínico correspondía a un adulto joven que evolucionó con una probable reacción paradojal, entidad más frecuente en la forma infanto-juvenil, su clínica inicial no era la característica, ya que en ella predomina la ausencia de manifestaciones respiratorias. Por otra parte, se descartaron causas de inmunocompromiso grave, como cáncer o infección por $\mathrm{VIH}$. En contexto de descarte de otra inmunosupresión diferente a esta última, se solicitó recuento de células T CD4+ con un valor de 952 (32\%), dentro de los parámetros de normalidad.

Esta presentación nos hace plantear probable enfermedad causada por $P$. lutzii. Si bien es un agente con su epicentro localizado en la región centro-oeste de Brasil, al tratarse de una especie más reciente aún faltan más estudios epidemiológicos y de diagnóstico específico para excluir este agente en nuestra región.

La frecuencia de afectación del SNC en PCM es variable, reportada entre un $10-27 \%$. Este compromiso es secundario a un foco primario, donde el hongo se disemina principalmente a través de la vía hematógena y/o linfática (6). El compromiso a nivel de SNC puede presentarse como única forma de manifestación de la enfermedad o con evidencia clínica a otro nivel, como los casos descriptos. En uno de los casos expuestos estaba presente el compromiso inicial mucocutáneo que facilitó el diagnóstico por obtención de una muestra menos invasiva.

La presentación pseudotumoral ocurre en el $80 \%$ de los pacientes. Los síntomas neurológicos dependen del sitio de compromiso del SNC. La hipertensión intracraneal y la ataxia de la marcha son los síntomas más reportados, como se presentaba en uno de los casos clínicos, ubicando al cerebelo como sitio preferencial de las lesiones (9).

Los hallazgos más frecuentes en la TC de encéfalo incluyen lesiones redondeadas, de localización variable, con edema perifocal, discreto efecto de masa y captación de contraste en anillo. La RM manifiesta lesiones iso/hipointensas en $\mathrm{T} 1$, hipointensas en T2, con edema periférico y realce anular con contraste.

En los diagnósticos diferenciales se incluyen principalmente enfermedades granulomatosas de otra etiología y procesos expansivos del SNC como abscesos, gliomas o metástasis, especialmente en individuos de áreas endémicas o que han visitado estas regiones. De esta manera, como presentamos en el segundo caso, por ausencia de disminución en el tamaño de la lesión en la RM control y un correcto tratamiento antifúngico, se recurrió a un procedimiento invasivo como la biopsia cerebral para evitar pasar por alto otra comorbilidad asociada.

Ambos casos clínicos remarcan que ante sospecha de PCM acompañado de signo-sintomatología neurológica es imperioso el estudio del posible compromiso del SNC. Debido a la alta sensibilidad de la RM de encéfalo para descartar afectación meníngea y parenquimatosa, ésta no debe postergarse y siempre debe realizarse tempranamente si se sospecha neuroparacoccidioidomicosis (NPCM). Aunque estas imágenes pueden orientar, su diagnóstico preciso está determinado por la detección o aislamiento del hongo en muestras provenientes de lesiones cerebrales o en LCR.

Considerando la morbilidad relacionada con procedimientos invasivos de este tipo, topografía de las lesiones que impidan su acceso o la falta de recursos para realizarlos de manera precoz, una herramienta serológica precisa basada en la detección de antígenos o anticuerpos contra el hongo es necesaria para mejorar el diagnóstico. 
De las pruebas serológicas actualmente disponibles para el diagnóstico de la enfermedad, la inmunodifusión sigue siendo la técnica más utilizada por su elevada sensibilidad ( $>90 \%$ ), fácil realización y bajo costo. Aunque la fijación de complemento también presenta buena sensibilidad, su uso es limitado por la baja especificidad. Ambas pruebas serológicas detectan anticuerpos contra componentes de Paracoccidioides y son útiles para el seguimiento de los pacientes $(10,8)$.

Los antígenos empleados para arribar al diagnóstico fueron los producidos a partir de la cepa B-339; no todas las especies de Paracoccidiodes producen este antígeno, lo que limita la eficiencia del diagnóstico serológico.

Durante el manejo de la PCM es importante mencionar no sólo fármacos antifúngicos, sino tener presente las comorbilidades que pueden actuar como factores de riesgo y las posteriores secuelas.

Estas últimas están presentes en más de un $60 \%$ de los pacientes con predominio de las alteraciones anatómicas y funcionales a nivel pulmonar. La más observada es la fibrosis pulmonar, descrita en el $50 \%$ de los pacientes con evolución, en menor porcentaje la enfermedad pulmonar obstructiva crónica, como se describe en uno de los casos clínicos.

P. brasilensis es sensible a la mayoría de los agentes disponibles. Sin embargo, cuando nos referimos específicamente al compromiso del SNC las alternativas se reducen a TMS, por su excelente paso a través de la barrera hematoencefálica; por este motivo se considera como primera opción terapéutica, con ventajas en cuanto a baja toxicidad, fácil administración vía endovenosa y posibilidad de pasaje a vía oral. En el segundo caso presentado, si bien se evidenciaba compromiso del SNC, se comenzó con anfotericina B ya que no había certeza que dicha lesión fuera secundaria a NPCM. Posterior a confirmación diagnóstica por biopsia se continuó con TMS en monoterapia.

Diversos trabajos también plantean la anfotericina B como buena alternativa terapéutica, reservándose para aquellos pacientes con intolerancia, resistencia o contraindicaciones para recibir sulfonamidas.

Otras opciones terapéuticas son los derivados azólicos, entre ellos el itraconazol, con amplias tasas de respuesta en el tratamiento de PCM sistémica. Sin embargo, debido a su escasa penetración en el SNC, no se recomienda su uso como terapia de primera línea. Como aprendizaje de uno de los casos clínicos es importante realizar una nueva neuroimagen, para descartar compromiso cerebral ante la ausencia de mejoría postratamiento y corrección de la hiponatremia, asociada como determinante de la clínica presente.

En formas graves es necesario un tratamiento prolongado, en general, con un mínimo de dos años. El tratamiento intravenoso puede continuar vía oral cuando existe mejoría clínica y garantía de correcta absorción digestiva. El mismo se debe continuar hasta la resolución de los signos clínico-radiológicos, con disminución significativa de los títulos de anticuerpos.

A pesar de una terapia antifúngica adecuada, un $6-30 \%$ de los pacientes pueden presentar un empeoramiento clínico-radiológico descripto como "reacción paradojal". Este fenómeno corresponde a la exacerbación de los síntomas clínicos o empeoramiento radiológico de las lesiones preexistentes o aparición de nuevas. Si bien no significa un fracaso de la terapia y, por ende, no se necesitan cambios de la misma, puede requerir el agregado de corticoesteroides como metilprednisona $1 \mathrm{mg} / \mathrm{kg} /$ día durante 4-6 semanas o mientras persista la clínica compatible $(11,12)$.

El momento de aparición de esta reacción es variable, desde dos semanas del inicio del tratamiento hasta varios meses luego de finalizado. El caso clínico presentado fue interpretado como una "reacción paradojal" por mostrar deterioro clínico a pesar de un diagnóstico preciso y una terapéutica adecuada, por lo que se iniciaron corticoides con buena respuesta.

Si bien el diagnóstico de NPCM sigue siendo difícil, la presentación clínica, junto con los datos epidemiológicos y de laboratorio deben ser analizados en conjunto para lograr un diagnóstico correcto.

Sin evidencias de compromiso sistémico, el diagnóstico suele ser tardío y con un consiguiente pobre pronóstico. Considerar tempranamente a la NPCM en los diagnósticos diferenciales puede revertir este escenario desfavorable. Los casos presentados pretenden mantener el ejercicio continuo del aprendizaje. 


\section{Referencias}

1 Canteros, Cristina. Paracoccidioidomicosis: crónica de una enfermedad olvidada. Revista Medicina. Volumen 78. Año 2018. №3.

2 Tichiellio, AG; Mangiaterra, M.; Giusiano, G. Paracoccidioidomicosis en la provincia de Formosa, Argentina. Rev Argent Microbiol. 2008; 40:24-9.

3 Maribel Choque Barrera, Eduardo Luis Suárez Barrientos, María Elena Calderón López. Paracoccidioidomicosis, apropos of a case. Hospital del Nino Manuel Ascencio Villarroel, Cochabamba, Bolivia. Médico Pediatra, Médico Pediatra Nutriólogo, Médico Pediatra Infectologa. Published online 2015; 38 (1): 30-33.

4 Shikanai-Yasuda MA, De Queiroz Telles Filho F, Pôncio Mendes R, Lopes Colombo A, Moretti ML. Consenso em paracoccidioidomicose. Revista da Sociedade Brasileira Medicina Tropical 2006; 39(3):297-310.

5 Vilela C, Pietra V. Paracoccidioidomicose com comprometimento do sistema nervoso central: revisão sistemática da literatura Revista da Sociedade Brasileira de Medicina Tropical. 2009; 42:691-7.

6 Calle D, Rosero D, Orozco L, Camargo D, Casteñeda E, Restrepo A. Paracoccidioidomycosis in Colombia: an ecological study. Epidemiol Infect. 2001; 126:309-15.

7 Central Nervous System Paracoccidioidomycosis: An Overview. The Brazilian Journal of Infectious Diseases 2005;9(2):126-133.

8 Mario León Silva-Vergara, Ivonete Helena Rocha, Rakel Rocha Vasconcelos, Andre'Luiz Maltos, Fernando de Freitas Neves, Luciana de Almeida Silva Teixeira, Delio Jose Mora. Central Nervous System Paracoccidioidomycosis in an AIDS Patient: Case Report. Mycopathologia (2014) 177: 137-141.

9 Restrepo A., McEwen J.G., Castaneda E. The habitat of Paracoccidioides brasiliensis: how far from solving the riddle? Medical Mycology 2001; 39: 233-411.
10 Pires de CZ, Fabiano de FM. Current knowledge on pathogenesis and inmunodiagnosis of Paracoccidioid-omycosis. Revita Iberoamericana de Micología 2000; 17: 41-8.

11 San-Blas G, Niño-Vega G, Iturriaga T. Paracoccidioides brasiliensis and paracoccidioidomycosis: molecular approaches to morphogenesis, diagnosis, epidemiology, taxonomy and genetics. Medicina Mycology 2002; 40: 225-242.

12 Ashbee HR, Barnes RA, Johnson EM, Richardson MD, Gorton R, Hope. Therapeutic drug monitoring (TDM) of antifungal agents: guidelines from the British Society for Medical Mycology. J Antimicrob Chemother. 2014 May; 69 (5):1162-76. Review. PubMed PMID: 24379304; PubMed Central PMCID: PMC3977608. 
NEUROPARACOCCIDIOIDOMYCOSIS:

TO KEEP IN MIND

\section{Report of cases}

SUMMARY: Paracoccidioidomycosis (PCM) is an endemic mycosis, from tropical and subtropical zones, with a higher prevalence in Latin America, produced by species of the genus Paracoccidioides. It is a deep chronic systemic mycosis, which mainly affects adult males. The involvement of the central nervous system (CNS) is described in 10-27\%. We present two cases with neurological manifestation upon admission.

Keywords: Paracoccidioidomycosis, neuroparacoccidioidomycosis, central nervous system 